\title{
Nonequilibrium molecular vibrons: An approach based on the nonequilibrium Green function technique and the self-consistent Born approximation
}

\author{
D. A. Ryndyk,* M. Hartung, and G. Cuniberti \\ Institut für Theoretische Physik, Universität Regensburg, D-93040 Germany
}

(Received 5 August 2005; published 18 January 2006)

\begin{abstract}
We consider the nonequilibrium quantum vibrations of a molecule clamped between two macroscopic leads in a current-carrying state at finite voltages. Our approach is based on the nonequilibrium Green function technique and the self-consistent Born approximation. Kinetic equations for the average populations of electrons and vibrons are formulated in the weak electron-vibron coupling case and self-consistent solutions are obtained. The effects of vibron emission and vibronic instability are demonstrated using few-orbital models. The importance of the electron-vibron resonance is shown.
\end{abstract}

DOI: 10.1103/PhysRevB.73.045420

PACS number(s): 85.65. $+\mathrm{h}, 73.23 .-\mathrm{b}, 73.63 . \mathrm{Kv}$

\section{INTRODUCTION}

During the past several years, nonequilibrium quantum transport in nanostructures and, in particular, transport through single molecules, has been the focus of both experimental and theoretical investigations because of possible electronic device applications. Recently, the interaction of electrons with molecular vibrations attracted attention following experiments on electron transport through single molecules. ${ }^{1-8}$ Theoretical treatments were presented in Refs. 9-21. In this paper, we consider a quantum theory of nonequilibrium vibronic excitation.

Basically there exist two main nonequilibrium effects; the electronic spectrum modification ${ }^{19,21}$ and the excitation of vibrons (quantum vibrations). ${ }^{12,13,18-20}$ In the weak electronvibron coupling case the spectrum modification is usually small (which is dependent, however, on the vibron dissipation rate, temperature, etc.) and the main possible nonequilibrium effect is the excitation of vibrons at finite voltages. We develop an analytical theory for this case. This theory is based on the self-consistent Born approximation (SCBA), which allows one to take easily into account and calculate nonequilibrium distribution functions of electrons and vibrons.

If the mechanical degrees of freedom are coupled strongly to the environment (dissipative vibron), then the dissipation of molecular vibrations is determined by the environment. However, if the coupling of vibrations to the leads is weak, we should consider the case when the vibrations are excited by the current flowing through a molecule, and the dissipation of vibrations is also determined essentially by the coupling to the electrons. In this paper, we show that the effects of vibron emission and vibronic instability are important especially in the case of electron-vibron resonances.

In the next section a simple model of an electron-vibron system is formulated. In Sec. III nonequilibrium Green functions and the Keldysh technique are used to obtain a full set of equations describing nonequilibrium electrons and vibrons self-consistently. Kinetic equations for the average populations of electrons and vibrons are formulated in the weak electron-vibron coupling case. In Sec. IV the solutions of these equations are obtained and the results are discussed.

\section{ELECTRON-VIBRON MODEL}

We describe a molecule coupled to free conduction electrons in the leads by a usual tunneling Hamiltonian. Furthermore, the electrons are coupled to vibrational modes. We do not consider the Coulomb interaction to avoid further effects, such as the Coulomb blockade and Kondo effect, which could dominate over the physics we want to address, however, self-consistent mean-field effects can easily be included in our approach. The full Hamiltonian is the sum of the molecular Hamiltonian $\hat{H}_{M}$, the Hamiltonians of the leads $\hat{H}_{R(L)}$, the tunneling Hamiltonian $\hat{H}_{T}$ describing molecule-lead coupling, the vibron Hamiltonian $\hat{H}_{V}$ including electron-vibron coupling, and the coupling of the vibrations to the environment

$$
\hat{H}=\hat{H}_{M}+\hat{H}_{L}+\hat{H}_{R}+\hat{H}_{T}+\hat{H}_{V} .
$$

A molecule (as well as a system of small quantum dots) is described by a set of localized states $|\alpha\rangle$ with energies $\epsilon_{\alpha}$ (tight-binding model) by the following model Hamiltonian:

$$
\hat{H}_{M}^{(0)}=\sum_{\alpha}\left[\epsilon_{\alpha}+e \varphi_{\alpha}(t)\right] d_{\alpha}^{\dagger} d_{\alpha}+\sum_{\alpha \neq \beta} t_{\alpha \beta} d_{\alpha}^{\dagger} d_{\beta}
$$

where $d_{\alpha}^{\dagger}, d_{\alpha}$ are the creation and annihilation operators in the states $|\alpha\rangle$, and $\varphi_{\alpha}(t)$ is the (self-consistent) electrical potential. The index $\alpha$ is used to mark the single-electron states including the spin degree of freedom.

The Hamiltonians of the right $(R)$ and left $(L)$ leads, shown in Fig. 1, are

$$
\hat{H}_{i=L(R)}=\sum_{k \sigma}\left(\epsilon_{i k \sigma}+e \varphi_{i}(t)\right) c_{i k \sigma}^{\dagger} c_{i k \sigma}
$$

$\varphi_{i}(t)$ are the electrical potentials of the leads, the index $k$ is the wave vector, but can be considered as representing another conserved quantum number, $\sigma$ is the spin index, but can be considered as a generalized channel number describing, e.g., different bands or subbands in semiconductors.

The tunneling Hamiltonian 


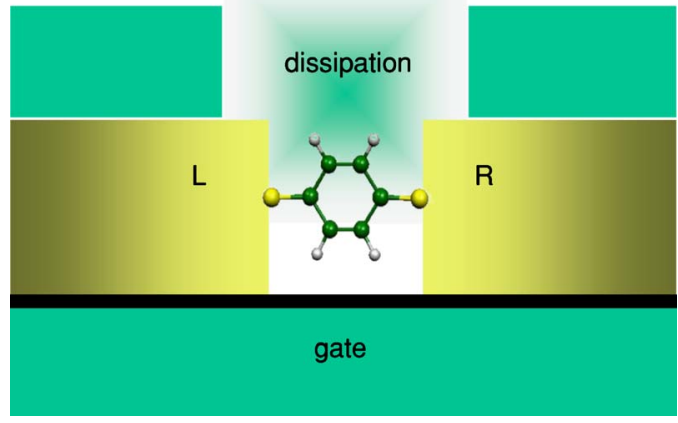

FIG. 1. (Color online) Schematic picture of the considered system.

$$
\hat{H}_{T}=\sum_{i=L, R} \sum_{k \sigma, \alpha}\left(V_{i k \sigma, \alpha} c_{i k \sigma}^{\dagger} d_{\alpha}+\text { H.c. }\right)
$$

describes hopping between the leads and the molecule. Direct hopping between two leads is neglected (the weak molecule-lead coupling case).

Vibrations and the electron-vibron coupling are described by the Hamiltonian ${ }^{14-16}$

$$
\hat{H}_{V}=\sum_{q} \hbar \omega_{q} a_{q}^{\dagger} a_{q}+\sum_{\alpha \beta} \sum_{q} M_{\alpha \beta}^{q}\left(a_{q}+a_{q}^{\dagger}\right) d_{\alpha}^{\dagger} d_{\beta} .
$$

Here vibrations are considered as localized phonons and $q$ is an index labeling them, not the wave vector. The first term describes the free vibrons with the energy $\hbar \omega_{q}$. The second term represents the electron-vibron interaction. We include both diagonal coupling, which describes a change of the electrostatic energy with the distance between atoms, and the off-diagonal coupling, which describes the dependence of the matrix elements $t_{\alpha \beta}$ over the distance between the atoms.

\section{NONEQUILIBRIUM SELF-CONSISTENT THEORY}

We use the nonequilibrium Green function (NGF) method, ${ }^{22,23}$ which now is a standard approach in mesoscopic physics and molecular electronics. ${ }^{1}$ We follow the formulation of Meir, Wingreen, and Jauho, ${ }^{24-26}$ which has already been applied to the case of self-consistency. ${ }^{21}$

The current in the left $(i=L)$ or right $(i=R)$ contact to the molecule is described by the well-known expression

$$
\begin{aligned}
J_{i=L, R}= & \frac{i e}{\hbar} \int \frac{d \boldsymbol{\epsilon}}{2 \pi} \operatorname{Tr}\left\{\boldsymbol { \Gamma } _ { i } ( \epsilon - e \varphi _ { i } ) \left(\mathbf{G}^{<}(\boldsymbol{\epsilon})\right.\right. \\
& \left.\left.+f_{i}^{0}\left(\boldsymbol{\epsilon}-e \varphi_{i}\right)\left[\mathbf{G}^{R}(\boldsymbol{\epsilon})-\mathbf{G}^{A}(\boldsymbol{\epsilon})\right]\right)\right\},
\end{aligned}
$$

where $f_{i}^{0}(\epsilon)$ is the equilibrium Fermi distribution function with the chemical potential $\mu_{i}$

$$
f_{i}^{0}(\epsilon)=\frac{1}{\exp \left(\left(\epsilon-\mu_{i}\right) / T\right)+1},
$$

and the level-width function is

$$
\boldsymbol{\Gamma}_{i=L(R)}(\boldsymbol{\epsilon})=\Gamma_{i \alpha \beta}(\boldsymbol{\epsilon})=2 \pi \sum_{k \sigma} V_{i k \sigma, \beta} V_{i k \sigma, \alpha}^{*} \delta\left(\boldsymbol{\epsilon}-\boldsymbol{\epsilon}_{i k \sigma}\right) .
$$

The matrix lesser (retarded, advanced) Green functions of a nonequilibrium molecule $\mathbf{G}^{<(R, A)} \equiv G_{\alpha \beta}^{<(R, A)}$ can be found from the Dyson-Keldysh equations in the integral form

$$
\begin{gathered}
\mathbf{G}^{R}(\boldsymbol{\epsilon})=\mathbf{G}_{0}^{R}(\boldsymbol{\epsilon})+\mathbf{G}_{0}^{R}(\boldsymbol{\epsilon}) \boldsymbol{\Sigma}^{R}(\boldsymbol{\epsilon}) \mathbf{G}^{R}(\boldsymbol{\epsilon}), \\
\mathbf{G}^{<}(\boldsymbol{\epsilon})=\mathbf{G}^{R}(\boldsymbol{\epsilon}) \boldsymbol{\Sigma}^{<}(\boldsymbol{\epsilon}) \mathbf{G}^{A}(\boldsymbol{\epsilon}),
\end{gathered}
$$

or from the corresponding equations in the differential form (see Ref. 21 and references therein).

Here $\boldsymbol{\Sigma}^{R,<}=\boldsymbol{\Sigma}_{L}^{R,<(T)}+\boldsymbol{\Sigma}_{R}^{R,<(T)}+\boldsymbol{\Sigma}^{R,<(V)}$, is the total selfenergy of the molecule composed of the tunneling (coupling to the left and right leads) self-energies $\boldsymbol{\Sigma}_{j=L, R}^{R,<(T)} \equiv \Sigma_{j \alpha \beta}^{R,<(T)}$ $=\sum_{k \sigma}\left\{V_{j k \sigma, \alpha}^{*} G_{j k \sigma}^{R,<} V_{j k \sigma, \beta}\right\}$, and the vibronic self-energy $\Sigma^{R,<(V)} \equiv \Sigma_{\alpha \beta}^{R,<(V)}$.

For the retarded tunneling self-energy $\boldsymbol{\Sigma}_{i}^{R(T)}$ one obtains

$$
\boldsymbol{\Sigma}_{i}^{R(T)}(\epsilon)=\Lambda_{i}\left(\epsilon-e \varphi_{i}\right)-\frac{i}{2} \boldsymbol{\Gamma}_{i}\left(\epsilon-e \varphi_{i}\right),
$$

where $\boldsymbol{\Lambda}_{i}$ is the real part of the self-energy, which usually can be included in the single-particle Hamiltonian $\hat{H}_{M}^{(0)}$, and $\boldsymbol{\Gamma}_{i}$ describes the level broadening due to the coupling to the leads. For the corresponding lesser function one finds

$$
\boldsymbol{\Sigma}_{i}^{<(T)}(\epsilon)=i \boldsymbol{\Gamma}_{i}\left(\epsilon-e \varphi_{i}\right) f_{i}^{0}\left(\epsilon-e \varphi_{i}\right) .
$$

In the standard self-consistent Born approximation, using the Keldysh technique, one obtains for the vibronic self-energies $^{13-16,18,19,26,27}$

$$
\begin{aligned}
\boldsymbol{\Sigma}^{R(V)}(\boldsymbol{\epsilon})= & \frac{i}{2} \sum_{q} \int \frac{d \omega}{2 \pi}\left(\mathbf{M}^{q} \mathbf{G}_{\epsilon-\omega}^{R} \mathbf{M}^{q} D_{q \omega}^{K}+\mathbf{M}^{q} \mathbf{G}_{\epsilon-\omega}^{K} \mathbf{M}^{q} D_{q \omega}^{R}\right. \\
& \left.-2 D_{q \omega=0}^{R} \mathbf{M}^{q} \operatorname{Tr}\left[\mathbf{G}_{\omega}^{<} \mathbf{M}^{q}\right]\right) \\
& \boldsymbol{\Sigma}^{<(V)}(\boldsymbol{\epsilon})=i \sum_{q} \int \frac{d \omega}{2 \pi} \mathbf{M}^{q} \mathbf{G}_{\epsilon-\omega}^{<} \mathbf{M}^{q} D_{q \omega}^{<}
\end{aligned}
$$

where $\mathbf{G}^{K}=\mathbf{2} \mathbf{G}^{<}+\mathbf{G}^{R}-\mathbf{G}^{A}$ is the Keldysh-Green function, and $\mathbf{M}^{q} \equiv M_{\alpha \beta}^{q}$.

If vibrons are noninteracting, in equilibrium, and nondissipative, then the vibronic Green functions write,

$$
\begin{gathered}
D_{0}^{R}(q, \omega)=\frac{1}{\omega-\omega_{q}+i 0^{+}}-\frac{1}{\omega+\omega_{q}+i 0^{+}} \\
D_{0}^{<}(q, \omega)=-2 \pi i\left[\left(f_{B}^{0}\left(\omega_{q}\right)+1\right) \delta\left(\omega+\omega_{q}\right)+f_{B}^{0}\left(\omega_{q}\right) \delta\left(\omega-\omega_{q}\right)\right],
\end{gathered}
$$

where the equilibrium Bose distribution function is

$$
f_{B}^{0}(\omega)=\frac{1}{\exp (\omega / T)-1} .
$$

In our model the retarded vibron function is calculated from the Dyson-Keldysh equation in the integral form

$$
D^{R}(q \omega)=D_{0}^{R}(q \omega)+D_{0}^{R}(q \omega) \Pi^{R}(q \omega) D^{R}(q \omega),
$$




$$
D^{R}(q, \omega)=\frac{2 \omega_{q}}{\omega^{2}-\omega_{q}^{2}-2 \omega_{q} \Pi^{R}(q, \omega)},
$$

where $\Pi(q, \omega)$ is the polarization operator (boson selfenergy). The equation for the lesser function (quantum kinetic equation in the differential form) is

$$
\left(\Pi_{q \omega}^{R}-\Pi_{q \omega}^{A}\right) D_{q \omega}^{<}-\left(D_{q \omega}^{R}-D_{q \omega}^{A}\right) \Pi_{q \omega}^{<}=0 .
$$

This equation, in the stationary case considered here, is algebraic in the frequency domain.

In the integral form one has the Keldysh equation for the lesser function

$$
D^{<}(q, \omega)=D^{R}(q, \omega) \Pi^{<}(q, \omega) D^{A}(q, \omega) .
$$

The polarization operator is the sum of two parts, environmental and electronic; $\Pi_{q \omega}^{R,<}=\Pi_{q \omega}^{R,<(\mathrm{env})}+\Pi_{q \omega}^{R,<(\mathrm{el})}$.

The environmental equilibrium part of the polarization operator can be approximated by the simple expressions

$$
\begin{gathered}
\Pi^{R(\mathrm{env})}(q, \omega)=-\frac{i}{2} \gamma_{q} \operatorname{sign}(\omega), \\
\Pi^{<(\mathrm{env})}(q, \omega)=-i \gamma_{q} f_{B}^{0}(\omega) \operatorname{sign}(\omega),
\end{gathered}
$$

where $\gamma_{g}$ is the vibronic dissipation rate, and $f_{B}^{0}(\omega)$ is the equilibrium Bose-Einstein distribution function.

The electronic contribution to the polarization operator within the SCBA is

$$
\Pi^{R(\mathrm{el})}(q, \omega)=-i \int \frac{d \epsilon}{2 \pi} \operatorname{Tr}\left(\mathbf{M}^{q} \mathbf{G}_{\epsilon}^{<} \mathbf{M}^{q} \mathbf{G}_{\epsilon-\omega}^{A}+\mathbf{M}^{q} \mathbf{G}_{\epsilon}^{R} \mathbf{M}^{q} \mathbf{G}_{\epsilon-\omega}^{<}\right),
$$

$$
\Pi^{<(\mathrm{el})}(q, \omega)=-i \int \frac{d \epsilon}{2 \pi} \operatorname{Tr}\left(\mathbf{M}^{q} \mathbf{G}_{\epsilon}^{<} \mathbf{M}^{q} \mathbf{G}_{\epsilon-\omega}^{>}\right) .
$$

We obtained the full set of equations, which can be used for numerical calculations. We simplify these equations and obtain some analytical results in the vibronic quasiparticle approximation, which assumes a weak electron-vibron coupling limit and a weak external dissipation of vibrons;

$$
\gamma_{q}^{*}=\gamma_{q}-2 \operatorname{Im} \Pi^{R}\left(\omega_{q}\right) \ll \omega_{q} .
$$

The spectral function of vibrons can be approximated by the Dirac $\delta$, and the lesser function reads

$$
D^{<}(q, \omega)=-2 \pi i\left[\left(N_{q}+1\right) \delta\left(\omega+\omega_{q}\right)+N_{q} \delta\left(\omega-\omega_{q}\right)\right],
$$

where $N_{q}$ is the (nonequilibrium) number of vibrations in the $q$ th mode. In this approximation the spectrum modification of vibrons is not taken into account, but the possible excitation of vibrations is described by the nonequilibrium $N_{q}$. The dissipation of vibrons is neglected in the spectral function, but is taken into account later in the kinetic equation for $N_{q}$. A similar approach to the single-level problem was considered recently in Refs. 13, 18, and 19. The more general case with the broadened equilibrium vibron spectral function does not seem to be very interesting, because in this case vibrons are not excited. Nevertheless, in the numerical calculation it can easily be taken into consideration.

From the general quantum kinetic equation for vibrons (20) we obtain in this limit

$$
N_{q}=\frac{\gamma_{q} N_{q}^{0}-\operatorname{Im} \Pi^{<}\left(\omega_{q}\right)}{\gamma_{q}-2 \operatorname{Im} \Pi^{R}\left(\omega_{q}\right)} .
$$

This expression describes the number of vibrons, $N_{q}$, in a nonequilibrium state, $N_{q}^{0}=f_{B}^{0}\left(\omega_{q}\right)$ is the equilibrium number of vibrons. In the linear approximation the polarization operator is independent of $N_{q}$ and $-2 \operatorname{Im} \Pi^{R}\left(\omega_{q}\right)$ describes an additional dissipation. Note that in equilibrium $N_{q} \equiv N_{q}^{0}$ because $\operatorname{Im} \Pi^{<}\left(\omega_{q}\right)=2 \operatorname{Im} \Pi^{R}\left(\omega_{q}\right) f_{B}^{0}\left(\omega_{q}\right)$. See also, the detailed discussion of vibron emission and absorption rates in Ref. 18.

For weak electron-vibron coupling the number of vibrons is close to equilibrium and is changed because of the vibron emission by nonequilibrium electrons, $N_{q}$, is roughly proportional to the number of such electrons, and the distribution function of the nonequilibrium electrons is essentially not changed by the interaction with vibrons (perturbation theory can be used). The situation changes, however, if the nonequilibrium dissipation $-2 \operatorname{Im} \Pi^{R}\left(\omega_{q}\right)$ is negative. In this case the number of vibrons can be essentially larger than in the equilibrium case (vibronic instability), and the change of the electron distribution function should be taken into account self-consistently.

In the stationary state the nonlinear dissipation rate

$$
\gamma_{q}^{*}=\gamma_{q}-2 \operatorname{Im} \Pi^{R}\left(\omega_{q}\right)
$$

is positive, but the nonequilibrium contribution to dissipation $-2 \operatorname{Im} \Pi^{R}\left(\omega_{q}\right)$ remains negative.

Additionally, to the vibronic quasiparticle approximation, the electronic quasiparticle approximation can be used when the coupling to the leads is weak. In this case the lesser function can be parametrized through the number of electrons $F_{\eta}$ in the eigenstates of the noninteracting molecular Hamiltonian $H_{M}^{(0)}$,

$$
G_{\alpha \beta}^{<}=i \sum_{\gamma \eta} A_{\alpha \gamma} S_{\gamma \eta} F_{\eta} S_{\eta \beta}^{-1},
$$

we introduce the unitary matrix $\mathbf{S}$, which transfers the Hamiltonian $\mathbf{H} \equiv H_{M \alpha \beta}^{(0)}$ into the diagonal form $\widetilde{\mathbf{H}}=\mathbf{S}^{-1} \mathbf{H S}$, so that the spectral function of this diagonal Hamiltonian is

$$
\widetilde{A}_{\delta \eta}(\epsilon)=2 \pi \delta\left(\epsilon-\tilde{\epsilon}_{\delta}\right) \delta_{\delta \eta},
$$

where $\tilde{\boldsymbol{\epsilon}}_{\delta}$ are the eigenenergies.

Note that in the calculation of the self-energies and polarization operators we cannot use the $\delta$-approximation for the spectral function (this is too rough and results in the absence of interaction out of the exact electron-vibron resonance). So that in the calculation we use actually Eq. (30) or

$$
\widetilde{G}_{\eta \delta}^{<}=i \widetilde{A}_{\eta \eta} F_{\eta} \delta_{\eta \delta}
$$

with a broadened equilibrium spectral function. This approximation can be systematically improved by including nonequilibrium corrections to the spectral function, which 
are important near the resonance. It is important to comment that for stronger electron-vibron coupling vibronic sidebands are observed in the spectral function and voltage-current curves at energies $\widetilde{\boldsymbol{\epsilon}}_{\delta} \pm n \omega_{q}$. We do not consider these effects in the rest of our paper and concentrate on resonance effects.

After corresponding calculations we finally obtain

$$
N_{q}=\frac{\gamma_{q} N_{q}^{0}-\sum_{\eta \delta} \kappa_{\eta \delta}\left(\omega_{q}\right) F_{\eta}\left(F_{\delta}-1\right)}{\gamma_{q}-\sum_{\eta \delta} \kappa_{\eta \delta}\left(\omega_{q}\right)\left(F_{\eta}-F_{\delta}\right)},
$$

where coefficients $\kappa_{\eta \delta}$ are determined by the spectral function and electron-vibron coupling in the diagonal representation

$$
\begin{gathered}
\kappa_{\eta \delta}\left(\omega_{q}\right)=\int \frac{d \epsilon}{2 \pi} \tilde{M}_{\eta \delta}^{q} \widetilde{A}_{\delta \delta}\left(\epsilon-\omega_{q}\right) \tilde{M}_{\delta \eta}^{q} \widetilde{A}_{\eta \eta}(\epsilon), \\
F_{\eta}=\frac{\widetilde{\Gamma}_{L \eta \eta} f_{L \eta}^{0}+\widetilde{\Gamma}_{R \eta \eta} f_{R \eta}^{0}+\sum_{q \eta}\left[\zeta_{\eta \delta}^{q} F_{\delta} N_{q}+\zeta_{\eta \delta}^{+q} F_{\delta}\left(1+N_{q}\right)\right]}{\widetilde{\Gamma}_{L \eta \eta}+\widetilde{\Gamma}_{R \eta \eta}+\sum_{q \eta}\left[\zeta_{\eta \delta}^{q}\left(1-F_{\delta}+N_{q}\right)+\zeta_{\eta \delta}^{+q}\left(F_{\delta}+N_{q}\right)\right]},
\end{gathered}
$$

$$
\zeta_{\eta \delta}^{ \pm q}=\tilde{M}_{\eta \delta}^{q} \tilde{A}_{\delta \delta}\left(\tilde{\epsilon}_{\eta} \pm \omega_{q}\right) \tilde{M}_{\delta \eta}^{q},
$$

here $\widetilde{\Gamma}_{i \eta \eta}$ and $f_{i \eta}^{0}$ are the level-width matrix in the diagonal representation and Fermi function at energy $\tilde{\epsilon}_{\eta}-e \varphi_{i}$.

These kinetic equations are similar to the usual golden rule equations, but are more general.

\section{RESULTS AND DISCUSSION}

Now let us consider several examples of vibron emission and vibronic instability.

(i) Vibron emission First we consider the most simple case, when the instability is not possible and only vibron emission takes place. This corresponds to a negative imaginary part of the electronic polarization operator; $\operatorname{Im} \Pi^{(R)}$ $\times\left(\omega_{q}\right)<0$. From Eq. (34) one can see that for any two levels with the energies $\tilde{\boldsymbol{\epsilon}}_{\eta}>\widetilde{\boldsymbol{\epsilon}}_{\delta}$ the coefficient $\kappa_{\eta \delta}$ is larger than $\kappa_{\delta \eta}$, because the spectral function $\widetilde{A}_{\delta \delta}(\epsilon)$ has a maximum at $\epsilon=\widetilde{\epsilon}_{\delta}$. The contribution of $\kappa_{\eta \delta}\left(\omega_{q}\right)\left(F_{\eta}-F_{\delta}\right)$ is negative if $F_{\eta}<F_{\delta}$. This takes place in equilibrium, and in nonequilibrium for transport through the symmetric molecules when higher energy levels are populated after lower levels. The example of such a system is shown in Fig. 2. Here we consider a simple three-level system $\left(\tilde{\epsilon}_{1}=1, \tilde{\epsilon}_{2}=2, \widetilde{\epsilon}_{3}=3\right)$ coupled symmetrically to the leads $\left(\Gamma_{L \eta}=\Gamma_{R \eta}=0.01\right)$. The current-voltage curve is the same with and without vibrations in the case of symmetrical coupling to the leads and in the weak electron-vibron coupling limit (if we neglect the changes in the spectral function). The figure shows how vibrons are excited and the number of vibrons $N_{V}$ in the mode with a frequency $\omega_{0}$ is presented in two cases. In the offresonant case (green triangles) $N_{V}$ is very small compared with the resonant case $\left(\omega_{0}=\widetilde{\epsilon}_{2}-\widetilde{\epsilon}_{1}\right.$, red crosses, the vertical scale is changed for the off-resonant points). In fact, if the number of vibrons is very large, the spectral function and the

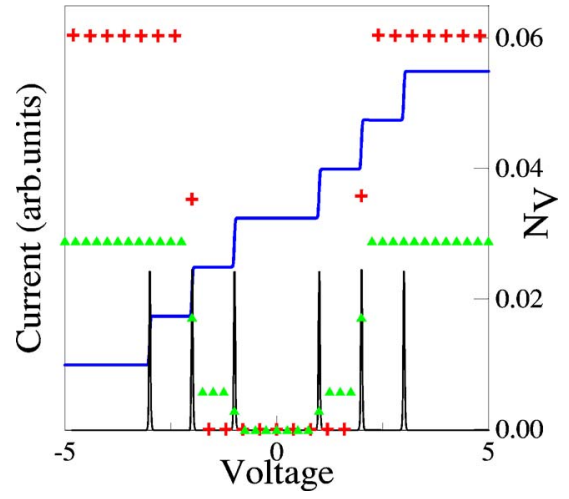

FIG. 2. (Color online) Vibronic emission in the symmetric multilevel model; voltage-current curve, differential conductance, and the number of excited vibrons in the off-resonant (triangles) and resonant (crosses) cases (for details, see the text).

voltage-current curve are changed. We shall consider this in a separate publication.

(ii) Vibronic instability Now let us consider the situation when the imaginary part of the electronic polarization operator can be positive; $\operatorname{Im} \Pi^{R}\left(\omega_{q}\right)>0$. Above we considered the normal case when the population of higher energy levels is smaller than lower levels. The opposite case $F_{2}>F_{1}$ is known as inversion in laser physics. Such a state is unstable if the total dissipation $\gamma_{q}^{*}$, Eq. (29), is negative, which is possible only in the nonstationary case. As a result of the instability, a large number of vibrons is excited, and in the stationary state $\gamma_{q}^{*}$ is positive. This effect can be observed for transport through asymmetric molecules, when higher energy levels are populated before the lower ones. The example of a such system is shown in Fig. 3. It is the same three-level system as before, but the first and second levels are not coupled symmetrically to the leads $\left(\Gamma_{L 1}=0.001, \Gamma_{R 1}=0.1\right.$, $\left.\Gamma_{L 2}=0.1, \Gamma_{R 2}=0.001\right)$. The vibron couples resonantly to these levels $\left(\omega_{q}=\tilde{\epsilon}_{2}-\tilde{\epsilon}_{1}\right)$. The result is qualitatively different from the symmetrical case. The voltage-current curve is now asymmetric and a large step corresponds to the resonant level with an inverted population.

Note the importance of the off-diagonal electron-vibron coupling for the resonant effects. If the matrix $\tilde{\mathbf{M}}$ in the

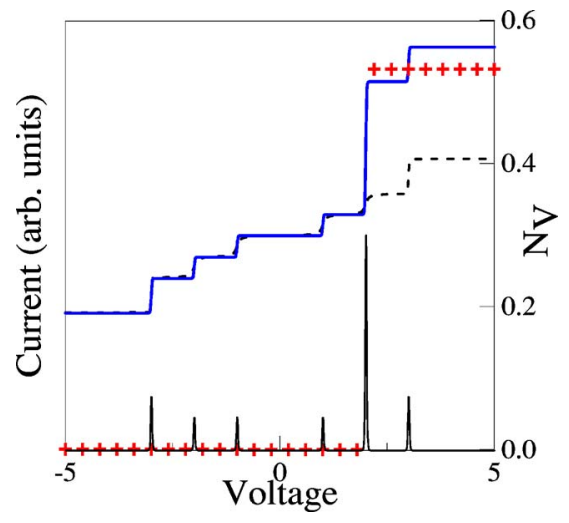

FIG. 3. (Color online) Vibronic instability in an asymmetric multilevel model; voltage-current curve, differential conductance, and the number of excited vibrons (crosses). The dashed line shows the voltage-current curve without vibrons (for details, see the text). 


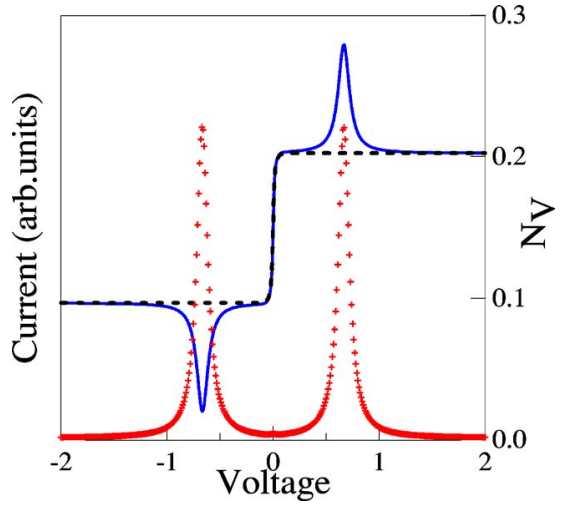

FIG. 4. (Color online) Floating level resonance; voltage-current curve and the number of excited vibrons (crosses). The dashed line shows the voltage-current curve without vibrons (for details, see the text).

eigenstate representation is diagonal, there is no resonant coupling between different electronic states.

(iii) Floating-level resonance Finally, let us consider the important case, when an initially symmetric molecule becomes asymmetric when the external voltage is applied. The reason for such asymmetry is simply that in the external electric field left and right atoms feel different electrical potentials and the position of the levels $\epsilon_{\alpha}=\epsilon_{\alpha}^{(0)}+e \varphi_{\alpha}$ is changed (float) with the external voltage. The example of a such system is shown in Fig. 4. Here we consider a two-level system, one level is coupled electrostatically to the left lead $\widetilde{\epsilon}_{1} \propto \varphi_{L}$, the other level to the right lead $\widetilde{\epsilon}_{2} \propto \varphi_{R}$. The tunneling coupling to the leads also is not symmetrical $\left(\Gamma_{L 1}=0.1, \Gamma_{R 1}\right.$ $\left.=0.001, \Gamma_{L 2}=0.001, \Gamma_{R 2}=0.1\right)$. The frequency of the vibration, coupling these two states, is $\omega_{0}=1$. When we sweep the voltage, a peak in the voltage-current curve is observed when the energy difference $\widetilde{\epsilon}_{1}-\widetilde{\epsilon}_{2} \propto \mathrm{eV}$ is going through the resonance $\widetilde{\epsilon}_{1}-\widetilde{\epsilon}_{2} \approx \omega_{0}$.

\section{CONCLUSION}

In conclusion, we considered the excitations of quantum molecular vibrations in the nonequilibrium state and their influence on the voltage-current curves of a single molecule placed between two equilibrium leads. The importance of vibron emission and vibronic instability in molecular transport is demonstrated.

\section{ACKNOWLEDGMENTS}

We thank J. Keller and K. Richter for valuable discussions. This work was supported by the Volkswagen Foundation under Grant No. I/78 340 and by the EU under Contract IST-2001-38951.
*On leave from the Institute for Physics of Microstructures, RAS, Nizhny Novgorod, Russia.

${ }^{1}$ Introducing Molecular Electronics, edited by G. Cuniberti, G. Fagas, and K. Richter, Lecture Notes in Physics Vol. 680 (Springer, Berlin, 2005).

${ }^{2}$ M. A. Reed, C. Zhou, C. J. Muller, T. P. Burgin, and J. M. Tour, Science 278, 252 (1997).

${ }^{3}$ H. Park, J. Park, A. K. L. Lim, E. H. Anderson, A. P. Alivisatos, and P. L. McEuen, Nature (London) 407, 57 (2000).

${ }^{4}$ J. Park, A. N. Pasupathy, J. I. Goldsmit, C. Chang, Y. Yaish, J. R. Petta, M. Rinkoski, J. P. Sethna, H. D. Abrun, and P. L. McEuen, Nature (London) 417, 722 (2002).

${ }^{5}$ W. Liang, M. P. Shores, M. Bockrath, J. R. Long, and H. Park, Nature (London) 417, 725 (2002).

${ }^{6}$ R. H. M. Smit, Y. Noat, C. Untiedt, N. D. Lang, M. C. van Hemert, and J. M. van Ruitenbeek, Nature (London) 419, 906 (2002).

${ }^{7}$ N. B. Zhitenev, H. Meng, and Z. Bao, Phys. Rev. Lett. 88, 226801 (2002).

${ }^{8}$ L. H. Yu and D. Natelson, Nano Lett. 4, 79 (2004); L. H. Yu, Z. K. Keane, J. W. Ciszek, L. Cheng, M. P. Stewart, J. M. Tour, and D. Natelson, Phys. Rev. Lett. 93, 266802 (2004).

${ }^{9}$ U. Lundin and R. H. McKenzie, Phys. Rev. B 66, 075303 (2002).

${ }^{10}$ J.-X. Zhu and A. V. Balatsky, Phys. Rev. B 67, 165326 (2003).

${ }^{11}$ S. Braig and K. Flensberg, Phys. Rev. B 68, 205324 (2003).

${ }^{12}$ V. Aji, J. E. Moore, and C. M. Varma, cond-mat/0302222 (unpublished).

${ }^{13}$ A. Mitra, I. Aleiner, and A. J. Millis, Phys. Rev. B 69, 245302 (2004).
${ }^{14} \mathrm{~T}$. Frederiksen, Master thesis, Technical University of Denmark (2004).

${ }^{15}$ T. Frederiksen, M. Brandbyge, N. Lorente, and A.-P. Jauho, Phys. Rev. Lett. 93, 256601 (2004).

${ }^{16}$ M. Hartung, Master thesis, University of Regensburg, 2004.

${ }^{17}$ M. Cízek, M. Thoss, and W. Domcke, Phys. Rev. B 70, 125406 (2004).

${ }^{18}$ T. Mii, S. G. Tikhodeev, and H. Ueba, Surf. Sci. 493, 63 (2001); 502, 26 (2002); Phys. Rev. B 68, 205406 (2003); S. G. Tikhodeev and H. Ueba, ibid. 70, 125414 (2004).

${ }^{19}$ M. Galperin, M. A. Ratner, and A. Nitzan, Nano Lett. 4, 1605 (2004); J. Phys. Chem. B 121, 11965 (2004); Nano Lett. 5, 125 (2005).

${ }^{20}$ J. Koch and F. von Oppen, Phys. Rev. Lett. 94, 206804 (2005); J. Koch, M. Semmelhack, F. von Oppen, and A. Nitzan, cond-mat/ 0504095 (unpublished).

${ }^{21}$ D. A. Ryndyk and J. Keller, Phys. Rev. B 71, 073305 (2005).

${ }^{22}$ L. Kadanoff and G. Baym, Quantum Statistical Mechanics (Benjamin, New York, 1962).

${ }^{23}$ L. V. Keldysh, Zh. Eksp. Teor. Fiz. 47, 1515 (1964), [Sov. Phys. JETP 20, 1018 (1965)].

${ }^{24}$ Y. Meir and N. S. Wingreen, Phys. Rev. Lett. 68, 2512 (1992).

${ }^{25}$ A.-P. Jauho, N. S. Wingreen, and Y. Meir, Phys. Rev. B 50, 5528 (1994).

${ }^{26}$ H. Haug and A.-P. Jauho, Quantum Kinetics and Optics of Semiconductors, Springer Series in Solid-State Sciences Vol. 123 (Springer, Berlin, 1996).

${ }^{27}$ J. Rammer and H. Smith, Rev. Mod. Phys. 58, 323 (1986). 